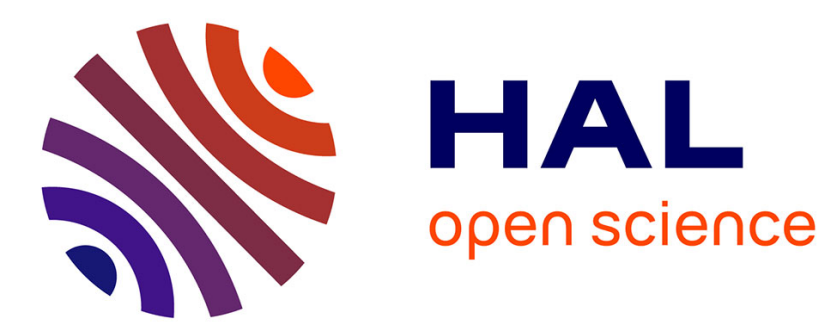

\title{
Organismes de formation et alternance Jean-Jacques Paul
}

\section{To cite this version:}

Jean-Jacques Paul. Organismes de formation et alternance. Formation Emploi. Revue française de sciences sociales, 1984, 7, pp.41-49. halshs-03184123

\section{HAL Id: halshs-03184123 \\ https://shs.hal.science/halshs-03184123}

Submitted on 4 Jun 2021

HAL is a multi-disciplinary open access archive for the deposit and dissemination of scientific research documents, whether they are published or not. The documents may come from teaching and research institutions in France or abroad, or from public or private research centers.
L'archive ouverte pluridisciplinaire HAL, est destinée au dépôt et à la diffusion de documents scientifiques de niveau recherche, publiés ou non, émanant des établissements d'enseignement et de recherche français ou étrangers, des laboratoires publics ou privés.

\section{(이) $\$$}

Distributed under a Creative Commons Attribution - NonCommercial - NoDerivatives| 4.0 


\title{
ORGANISMES DE FORMATION ET ALTERNANCE
}

\author{
par Jean-Jacques Paul
}

L'alternance apparaît aux yeux de beaucoup comme un procédé pédagogique novateur particulièrement bien adapté à un public qui a quitté l'école en situation d'échec. Néanmoins, les enjeux qui lui sont liés sont multiples, tant en ce qui concerne ses potentialités pédagogiques qu'en ce qui a trait aux risques de voir s'institutionnaliser une nouvelle catégorie de main-d'œuvre constituée des jeunes stagiaires.

Dans cette optique, le questionnement privilégié ici consistera à tenter de repérer la manière dont les organismes de formation ont incorporé l'alternance dans leur pédagogie et dans quelle mesure a pu naître une synergie entre ceux-ci et les entreprises à travers la définition et it. déroulement des stages.

Compte tenu du peu de recul que l'on a encore par raprort au dispositif mis en vigueur en 1982 et de la fragilité de nos observations, il nous a semblé préférable de nous en tenir, pour le moment, à une présentation empirique qui, si elle n'est probablement pas neutre sur le plan théorique, n'entend pas donner un caractère intangible aux observations relevées.

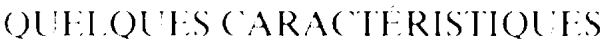 I)HSOR(IANISMES DEF FORMATION FI IDI I.IUL PUBI.IC}

Pour essayer de comprendre la position des organismes par rapport à la gestion de l'alternance, il s'agit déjà de connaître leur rattachement institutionnel, que nous analyserons dans un premier temps à partir du cas de la Bourgogne. Puis nous examinerons quelques caractéristiques des formateurs en essayant de distinguer leur accoutumance à l'entreprise et leur sensibilité à l'alternance. Dans un troisième point, seront abordées rapidement l'origine sociale et scolaire des jeunes, dans la mesure où le jugement que l'on peut porter sur le déroulement des formations doit être resitué par rapport à celles-ci.

Les organismes de formation impliqués dans le dispositif

Pour comprendre quels sont les organismes qui ont participé à la première campagne en Bourgogne, analysons tout d'abord la répartition par type avant de nous consacrer à une analyse historique rapide.
Si nous considérons tout d'abord les nombres théoriques d'heures stagiaires, calculées à partir des listes des conventions agréées, il apparaît que l'Education nationale va occuper une place considérable, effectuant les deux cinquièmes des heures de stage. Mais il faut noter aussi le poids important des associations qui vont traiter $30 \%$ des heures. Les milieux professionnels seront surtout représentés par les chambres consulaires, qui dispensent $12 \%$ des heures, alors que les ASFO se cantonnent dans un rôle modeste, avec $2 \%$ des heures. D'autres organismes publics ou para-publics, comme l'AFPA ou les organismes rattachés au ministère de l'Agriculture, atteignent un pourcentage total de $15 \%$.

\section{RÉPARTITION DU NOMBRE D'HEURES-STAGIAIRES PAR TYPE D'ORGANISME DANS LE CADRE DU DISPOSI'TIF \\ ET DU PACTE NATIONAL POUR L'EMPLOI 1980-1981}

\begin{tabular}{|l|c|c|}
\hline \multicolumn{1}{|c|}{ Organisme } & $\begin{array}{c}\text { Nb heures stagiaires } \\
\text { actions « 16-18 } \\
\text { (pourcentages) }\end{array}$ & $\begin{array}{c}\text { Nb heures stagiaires } \\
\text { PNE 1980-81 } \\
\text { (pourcentages) }\end{array}$ \\
\hline Education & 1259430 & 238700 \\
nationale & $(40,5)$ & $(47,1)$ \\
Chambres & 370425 & 120660 \\
consulaires & $(11,9)$ & $(23,8)$ \\
ASFO & 70500 & 101880 \\
& $(2,3)$ & $(20,1)$ \\
Associations & 937075 & 29060 \\
Autres publics & $(30,1)$ & $(5,7)$ \\
et para-publics & 473055 & - \\
Autres privés & $(15,2)$ & $(3,3)$ \\
\hline Total & - & 506980 \\
\hline
\end{tabular}

Pour juger de cette répartition, nous pouvons, par exemple, la comparer avec celle concernant le nombre d'heures stagiaires dispensées dans le cadre du dernier Pacte National pour l'Emploi (PNE). Cette comparaison n'a pour but que d'insister sur les traits spécifiques des organismes impliqués dans le dispositif «16-18». Une analyse fine de la participation des organismes aux formations jeunes nécessiterait, en effet, l'inclusion des stages «18-21». 


\section{L'OBSERVATION ET L'EVALUATION DU DISPOSITIF DE FORMATION ET D'INSERTION DES JEUNES DE 16 A 18 ANS EN BOURGOGNE}

Concernant la première campagne, l'Institut de Recherche sur l'Economie de l'Education * a été chargé de mener l'observation et l'évaluation du dispositif sur la Bourgogne. Un rapport final a été présenté en août 1983.

Pour mener à bien ce travail, un certain nombre d'outils ont été utilisés. D'une part, ont été exploités les documents administratifs de type listes d'agrément des stages ou listes d'entreprises d'accueil et d'autre part, plusieurs ensembles d'enquêtes ont été réalisés.

Soixante-deux stages ont été analysés à partir d'un questionnaire traitant du processus d'élaboration du projet pédagogique, des objectifs de stage, du mode de recrutement des stagiaires, du rythme d'alternance, de la formation des formateurs.

Quatorze enquêtes ont été réalisées auprès d'un échantillon d'organismes impliqués dans le dispositif, visant à connaître leur histoire institutionnelle, l'origine et le montage des actions dans le cadre du dispositif ainsi que les moyens mis en œuvre.

L'accueil en entreprise a été étudié à partir de vingt-cinq enquêtes réalisées dans l'agglomération dijonnaise auprès de chefs d'entreprise ou de responsables du personnel et traitant de la politique de formation de l'entreprise, de ses relations avec l'organisme de formation maître-d'œuvre du stage, du déroulement du stage.

Enfin, les caractéristiques des jeunes ont été collectées à partir de l'exploitation de huit cents fiches individuelles. Cette exploitation a concerné les caractéristiques socio-économiques et éducatives des jeunes, l'examen du processus d'orientation, l'observation du suivi du stagiaire.

L'ensemble des observations est consigné dans le rapport "Observation et évaluation du dispositif de formation et d'insertion des jeunes de 16 a 18 ans en Bourgogne. Bilan d'une première année de fonctionnement ", réalisé par J. Danrey, A. Giffard, J.-J. Paul, M. Richard et disponible à l'IREDU.

* IREDU, Université de Dijon, BP 138, 21004 Dijon Cédex.

Notre présentation met en lumière deux phénomènes importants. D'une part, les organismes liés au secteur patronal ont beaucoup moins participé aux formations du dispositif qu'à celles des Pactes et, à l'inverse, la participation des associations s'est considérablement accrue. Il faut d'ailleurs signaler au passage que l'augmentation de la part des associations est notable à partir de la mise en place du Plan Avenir Jeunes, puisqu'elles ont effectué dans ce cadre, $19 \%$ des heures stagiaires.
Cette évolution n'est probablement pas neutre par rapport à l'utilisation de l'alternance dans la mesure où les organismes a priori les plus familiers de l'entreprise se sont effacés au cours de la première année de fonctionnement du dispositif.

Un point intéressant à noter concerne la structure interne du groupe des associations. On aurait peut-être facilement tendance à imaginer le développement de la participation du secteur associatif appuyé sur l'émergence de petites associations créées à l'occasion du dispositif ou sur la participation à la formation d'associations jusqu'alors étrangères à cette activité.

Or, si nous considérons les cinq associations les plus anciennes en matière de formation des jeunes sur la région, nous nous apercevons qu'elles assurent $56 \%$ des heures stagiaires dispensées par le secteur associatif. Dans le même ordre d'idée, nous pouvons noter la part modeste des missions locales et permanences d'accueil (1\% de l'ensemble des heures stagiaires), voire des municipalités $(4,3 \%$ de l'ensemble des heures stagiaires).

Certes, il ne faut pas nier l'entrée en jeu de nouveaux acteurs de formation dans le cadre du mouvement associatif, mais on doit avoir à l'esprit la place considérable des associations anciennes en matière de formation des jeunes.

Après ces caractéristiques d'ensemble des organismes impliqués dans le dispositif, intéressons-nous maintenant à la structure interne de ces organismes, en essayant en particulier de mettre en valeur les liens qu'ils entretiennent avec les entreprises.

L'ancienneté en matière de formation de nombreux organismes impliqués dans le dispositif qu'ont permis de repérer les précédentes données est confirmée par les enquêtes que nous avons effectuées au sein d'un certain nombre d'entre eux.

Ce qui apparaît intéressant, compte tenu de notre problématique, est que des contacts anciens existent entre les organismes de formation enquêtés et le milieu professionnel. Cette remarque va de soi pour les ASFO, associations de formation du secteur patronal, et pour les centres de formation de CCI. Mais elle vaut aussi pour les GRETA (groupements d'établissements d'enseignement), dont le conseil externe (conseil de la formation continue) comprend un collège patronal et un collège syndical. Et les associations engagées dans le dispositif se trouvent dans le même cas, les conseils d'administration comprenant toujours des représentants d'employeurs et de syndicats. Ainsi, l'une des associations enquêtées se définit-elle comme un organisme tripartite, son conseil d'administration étant composé d'un tiers d'employeurs, d'un tiers de syndicalistes, d'un tiers de représentants de 
la vie associative, la présidence étant assurée à tour de rôle par un employeur ou un syndicaliste.

La plupart des organismes engagés dans le dispositif ont donc des contacts soutenus avec le milieu professionnel, qui se traduisent souvent sur un plan institutionnel. Bien entendu, ces contacts puisent leurs origines dans la mise en place d'actions de formation continue de salariés $q u$ 'assurent ces organismes depuis plusieurs années. Et les associations ne sont pas étrangères à ce mouvement, comme les données précédentes le font remarquer et comme l'indique l'enquête directe auprès des organismes.

Les organismes de formation du dispositif, de par les liens souvent anciens tissés avec les entreprises, disposaient donc d'une structure apparemment propice au bon fonctionnement de l'alternance. Néanmoins, si le recours à l'alternance n'a pas eu les résultats escomptés, il semblerait que ce soit un phénomène pas tant lié à la structure institutionnelle des organismes qu'aux caractéristiques de formateurs et des jeunes, les partenaires du terrain, en quelque sorte.

\section{Qui sont les formateurs?}

L'expérience des formateurs est, bien entendu, un atout essentiel de la réussite d'une pédagogie de l'alternance. Cependant, force est de constater que l'alternance, en tant que processus de formation interactif entre formation en organisme et confrontation au réel en entreprise, et en outre appliqué à l'insertion sociale et professionnelle, apparaît comme un principe nouveau, qui avait été peu développé sur ces bases avant la mise en œuvre du dispositif. Il ne faut donc pas s'attendre à ce que les formateurs aient une expérience approfondie quant au recours pédagogique à l'alternance. Et leurs caractéristiques individuelles révèlent bien ce phénomène.

Avant de considérer les formateurs, arrêtons-nous un instant sur le cas des coordonnateurs des actions. Dans douze des quatorze organismes enquêtés, le coordonnateur fait partie de l'organisme. Dans deux cas seulement (un GRETA et une ASFO), le coordonnateur a été recruté, sur contrat, pour la durée de l'action.

Par contre, le recrutement de formateurs sur contrat a été une pratique plus fréquente, ayant concerné six organismes sur les quatorze rencontrés. I.e cas le plus remarquable a trait à une association qui comprend un effectif permanent de vingt-sept personnes et qui a recruté sur contrat à durée déterminée trente formateurs à temps plein et dix formateurs à temps partiel.

Mais la pratique la plus généralisée est celle qui consiste à faire appel à des vacataires rémunérés à l'heure. Cette stratégie se rencontre le plus intensément au sein des organismes intervenant fréquemment dans le cadre de la formation continue d'entreprise (GRETA, ASFO, établissements de formation rattachés aux chambres de commerce). Et, bien souvent, ce sont les mêmes formateurs auxquels l'organisme va faire appel pour la formation continue d'entreprise ou les actions " jeunes".

Les caractéristiques des formateurs s'orienteront donc selon les types d'organismes de rattachement, avec en corollaire à cette dernière dimension, le type d'action, insertion ou qualification. Ainsi, les formateurs recrutés sur contrat par les associations, souvent pour des actions d'insertion, se rattachent plutôt au monde des éducateurs et animateurs socio-culturels. Les organismes des milieux professionnels s'adresseront quant à eux de préférence à des intervenants vacataires ayant une expérience professionnelle et une formation spécialisée assez élevée.

On voit donc se dessiner une typologie asscz caricaturale mais qui traduit indiscutablement assez bien la réalité. On aurait ainsi d'un côté un groupe de formateurs professionnels en exercice, connaissant bien le monde du travail, mais habitués à un public d'adultes en formation continue, qui va se retrouver quelque peu déconcerté face à un public de jeunes au niveau scolaire faible, aux attentes floues, peu discipliné. D'un autre côté, on repère un groupe de formateurs sensibles aux problèmes de la population jeune, mais connaissant mal l'entreprise.

Bien sûr, c'est tout l'enjeu de l'alternance, son caractère subversif pourrait-on dire, que d'impliquer des formateurs dans une des dimensions de la formation qu'ils ignorent.

Ainsi, les formateurs, familiers de l'entreprise, vont devoir découvrir un public plus difficile que celui auquel ils ont coutume d'enseigner et utiliser les stages non seulement comme des périodes de mise en pratique de savoirs théoriques, mais aussi comme des clés d'insertion sur le monde du travail. De même, ils devront admettre que les stages auront un effet de retour non seulement sur les demandes de formation mais aussi sur les comportements. En outre, ces professionnels interviennent souvent à la vacation et auront rarement l'occasion d'assurer le suivi en entreprise.

Quant aux formateurs plus ouverts à l'approche socioéducative des jeunes, souvent connaissant peu le monde de l'entreprise, ils hésiteront à engager un dialogue pédagogique avec les professionnels, laissant ceux-ci conduire le stage sans consigne.

Ce sera aussi souvent le cas des formateurs des GRETA, encore marqués par le fonctionnement de l'enseignement initial.

Un dernier paramètre a aussi rendu peu facile la mise en œuvre de l'alternance. Il s'agit des caractéristiques socia- 
les et scolaires des jeunes. Nous allons nous arrêter un instant sur ce point.

\section{L'origine sociale et scolaire des jeunes}

Nous présenterons quelques données, essentiellement pour bien faire comprendre les difficultés des formateurs face à ce public.

L'origine sociale modeste n'est pas une surprise mais les chiffres sont quand même interpellateurs. Ainsi, $65 \%$ des jeunes sont issus de familles de quatre enfants et plus. Si une mère sur deux a une profession, ce sera dans trois cas sur cinq une profession exercée dans les services subalternes de la Santé, dans les services d'entretien et aux particuliers et, dans un cas sur cinq, une profession d'ouvrière ou d'employée. De même, $56 \%$ du public sont issus de familles dont le père est ouvrier, dans $43 \%$ des cas ouvrier qualifié et donc dans $57 \%$ des cas, ouvrier non qualifié. De plus, un phénomène doit retenir l'attention, à savoir que $20 \%$ des chefs de famille sont retraités ou invalides.

Et comme l'on pouvait s'y attendre, $80 \%$ des jeunes accueillis n'ont aucun diplôme et $53 \%$ d'entre eux n'ont pratiquement aucune formation professionnelle de base.

Ces caractéristiques vont donc être un des éléments importants de la difficulté des organismes à affronter le dispositif et, par là, à gérer l'alternance.

Bon nombre d'organismes rencontrés se sont, en effet, révélés surpris par le faible niveau scolaire des jeunes et ont dû réviser à la baisse leurs espérances en ce qui concerne en particulier les stages d'insertion. Selon une association, rien ne peut être proposé à $40 \%$ des jeunes à la fin des actions d'insertion, dans la mesure où il y a une profonde discordance entre les aptitudes des jeunes et celles exigées pour suivre une formation qualifiante.

Par ailleurs, plusieurs organismes estiment que les normes financières et organisationnelles ne permettent pas de s'occuper des jeunes qui ont le plus de difficultés, dans la mesure où il serait nécessaire de pouvoir s'adjoindre le concours d'un psychologue ou d'un phonéticien.

Néanmoins, l'analyse des contenus et de l'organisation de formations permet de penser que l'alternance n'est souvent restée qu'un principe que les organismes ont eu du mal à utiliser.

\section{I.A MISE EN PIACE DE I ALTERNANC 'E PAR IIES ORGANISMES IDE FORMATION}

La traduction du principe de l'alternance dans les faits a nécessité de la part des organismes de formation plu- sieurs étapes dans l'élaboration des stages. A des fins didactiques, nous avons distingué trois moments : celui qui concerne la conception du stage et de la pédagogie développée au centre de formation. celui qui a trait à la prise de contact avec les entreprises et enfin celui qui est lié à la définition pédagogique du stage en entreprise avec celle-ci.

\section{La fonction de l'alternance dans la pédagogie des organismes de formation}

Le premier constat qui s'impose est que les coordonnateurs, pour ne pas parler des formateurs, de par leur peu d'expérience et la faible dynamique créée avec les tuteurs, ou les responsables des entreprises, avouent ne pas avoir su faire jouer à plein l'alternance et peut-être, plus généralement, combiner les dimensions formation professionnelle et insertion sociale du dispositif.

Ceci se ressent bien à l'observation des objectifs de formation. Ainsi, au sein des stages d'insertion que nous avons observés (trente-et-un), on note une quasiabsence de l'objectif d'accession à un stage de qualification. Si pour certains organismes, le stage en entreprise est considéré comme le premier stade qui permet d'atteindre l'objectif d'une qualification, pour beaucoup, celui-ci constitue une activité qui n'a pas un rôle plus spécifique que n importe quel moment de formation.

En ce qui concerne les stages d'orientation, il est à noter que certains organismes comme les deux GRETA que nous avons rencontrés, s'appuient sur le stage en entreprise comme pivot (trois quarts du temps). Ce dernier s'effectue en trois périodes, dans des lieux différents à chaque fois. La découverte d'un métier ou d'une situation d'entreprise permet aux jeunes de se situer dans un cadre avant tout professionnel. Ce type d'expérience apparaît cependant assez exceptionnel.

Quant à l'alternance au sein des stages de qualification, elle a souvent permis aux jeunes de mettre en pratique leurs savoirs, mais bien souvent sans que se crée un processus interactif entre périodes en entreprises et périodes en organismes.

D'ailleurs, lorsque l'on aborde avec les formateurs le thème des méthodes pédagogiques, l'alternance n'apparaît pas. Peut-être ceci n'est-il pas étonnant lorsque l'on sait que souvent la pédagogic traditionnelle de l'enseignement initial est restée prégnante.

En effet, pour un tiers des organismes réalisant des stages d'insertion et pour la quasi-totalité des instituts de formation qui mettent en place des actions de qualification, la distribution des temps de formation au sein des emplois du temps est impérative. 
De plus, des phénomènes quantitativement analogues concernent le fait qu'un certain nombre d'organismes ne dispensent pas les contenus de formation dans le cadre d'une certaine globalité mais divisés entre le français, les maths, les sciences, la technologie...

Enfin, dans le cadre d'une action d'insertion et d'une de qualification, l'évaluation des acquis est réalisée à l'aide du traditionnel système de notation-contrôle de l'institution scolaire.

Finalement, le cumul de ces trois éléments amène à reproduire le système scolaire que ces mêmes organismes condamnent fortement.

Cette situation nous invite à considérer que la souplesse de fonctionnement institutionnel ou pédagogique offerte ne constitue pas toujours une condition suffisante au développement des capacités d'imagination de la part des formateurs, ou que cette réalité est la conséquence de l'inexpérience de la majorité d'entre eux, ces derniers cherchant, alors, à se sécuriser au moyen d'un cadre organisationnel rigide.

La prise en compte du rythme adopté pour l'alternance révèle elle-aussi les difficultés des coordonnateurs. En effet, si l'on considère que le principe de l'alternance se présente comme une répétition régulière de périodes, successivement consacrées aux stages en entreprise et aux phases de formation au sein de l'organisme, force est de constater que plusieurs actions, de qualification notamment, n'appliquent pas cette méthode. Il convient par ailleurs de remarquer la très grande diversité des rythmes d'alternance et de signaler la difficulté qu'éprouvent les coordonnateurs, lors des entretiens, à motiver les choix réalisés.

En définitive, si l'utilité de l'alternance paraît majoritairement acquise par les coordonnateurs des actions, les motifs des choix organisationnels effectués paraîssent souvent confus.

\section{La prise de contact avec les entreprises}

Les modalités selon lesquelles les organismes de formation ont pris contact avec les entreprises auront varié selon l'intensité des liens qui les reliaient.

Ainsi, dans le cas d'un stage organisé par le centre de formation d'une chambre de commerce, l'organisme a pu se contenter de contacter les entreprises par téléphone pour trouver les places de stage, dans la mesure où des contacts souvent anciens liaient celles-ci à l'organisme.

Dans le même ordre d'idée, en ce qui concerne un stage de qualification organisé par un GRETA, le formateur responsable a utilisé ses relations personnelles avec un certain nombre de garagistes pour trouver des places de stages.

A l'inverse, les entreprises que nous avons enquêtées, qui ont accueilli les stagiaires d'insertion d'une municipalité et d'une association, n'avaient jamais eu de contacts préalables avec ces organismes. Dans le premier cas, ce sont les stagiaires qui ont fait du démarchage, dans le second, l'association a téléphoné.

La recherche des lieux de stage par les stagiaires euxmêmes est présentée par les associations qui y ont recours, car c'est plutôt ce type d'organisme qui a privilégié ce mode de prise de contact avec les entreprises, comme un outil pédagogique permettant de développer l'autonomie du jeune et sa connaissance du milieu. Sans vouloir se prononcer sur l'éventuelle justification que peut représenter une telle attitude de la part de certains organismes face à leur méconnaissance du système productif, il est indéniable que le démarchage réalisé par les stagiaires s'est concentré sur les entreprises les plus facilement accessibles. Il s'est agi notamment des petites entreprises, en particulier du commerce et des services, ce qui a été l'une des causes de la sur-représentation des entreprises de ces catégories au sein du dispositif.

Ces modalités de prise de contact avec les entreprises, et les liens organismes-entreprises qui les sous-tendent. peuvent se traduire d'ailleurs en une typologie des entreprises que l'on peut construire à partir de leur degré et de leur mode d'implication dans le dispositif.

Nous pouvons distinguer à ce propos quatre sous-ensembles, dont on peut évaluer l'importance de chacun au sein de l'échantillon.

Le premier groupe est composé des entreprises actives et impliquées volontairement : six entreprises sur les vingtcinq. Il s'agit essentiellement d'une part d'administrations, et d'autre part d'établissements dont le caractère social, crèches, cliniques, les a conduits à accueillir des stagiaires et qui se considèrent comme terrains de stage.

Dans le cas des administrations, si les directives nationales ont pu jouer dans le processus de décision d'accueil des stagiaires, l'attitude de certains syndicalistes a été aussi un moteur important de l'engagement. Ainsi, certaines administrations ont-elles pris l'initiative du contact avec les organismes de formation.

Le second groupc cst constitué des entreprises que l'on pourrait qualifier "d'intéressées ", dont les qualifications auxquelles elles recourent majoritairement leur permettent de faire un usage productif des stagiaires. II s'agit en particulier du secteur commercial ; ce groupe représente un cinquième de notre échantillon. Elles auront vu les avantages qu'elles pouvaient retirer de l'accueil des stagiaires, et les auront reçus après un contact direct de la part de ceux-ci ou des organismes de formation. 
Le troisième groupe se caractérise plutôt par le fait que les entreprises qui le composent avaient déjà des relations avec les organismes, dans le cadre de l'apprentissage ou de l'embauche de jeunes issus de classes de CAP, et ont souvent été "entraînées ", non sans réticence, à accueillir des stagiaires. Ce sont souvent des entreprises de petite taille, de services marchands ou du secteur artisanal. On peut évaluer cet ensemble à huit entreprises sur les vingt-cinq de l'échantillon.

Quant au quatrième groupe, il est composé d'entreprises " indifférentes », qui ont accepté sans connaître le dispositif, d'accueillir des stagiaires après une visite de ceux-ci ou un contact informel de l'organisme de formation. Il comprend six entreprises sur les vingt-cinq.

Bien entendu, cette typologie est caricaturale dans la mesure où certaines entreprises pourraient se rattacher à plus d'un groupe, mais elle correspond assez fidèlement aux grandes tendances comportementales de celles-ci.

Un point à noter à propos de la prise de contact entre organismes et entreprises est que le dispositif a apparemment conduit bon nombre d'entreprises à ouvrir leurs portes à des stagiaires. Ainsi, au sein des vingt-cinq entreprises enquêtées, seules trois avaient déjà accueilli des stagiaires des Pactes, trois autres accueillant épisodiquement des stagiaires de formation initiale.

\section{La définition pédagogique du stage avec les entreprises}

Nous avons montré que les organismes de formation, de par la relative nouveauté du principe de l'alternance et la faible expérience des formateurs, avaient eu des difficultés à inclure l'alternance dans l'élaboration pédagogique du stage. Si les expériences des jeunes en stage ont été relativement peu prises en compte lors des périodes de regroupement, de même les organismes n'ont pas su définir avec les entreprises ce qu'ils attendaient d'elles, notamment par rapport aux savoirs à transmettre lors de ces périodes. Ceci est particulièrement flagrant lorsque l'on mène l'investigation au sein des entreprises qui ont accueilli des stagiaires.

Ce point est d'autant plus important que dans l'esprit des promoteurs du dispositif, l'organisme de formation devait avoir un rôle clé dans la définition des tâches à confier aux stagiaires et dans la dynamisation de l'alternance. Pour bien insister sur ce fait, et etı rupture avec l'organisation des Pactes, l'organisme de formation s'est vu confier dans les textes la responsabilité de l'ensemble de l'action de formation.

Les entreprises ont donc eu peu affaire aux organismes si ce n'est lors des contacts préliminaires, mais qui n'ont en général concerné que le placement au sens strict, et lors de visites rapides au cours du stage qui ont rarement été l'objet de discussions sur le contenu pédagogique de celui-ci.

Ainsi, il apparaît à travers les enquêtes que les chefs d'entreprise connaissent peu le dispositif en tant que tel et accueillent les stagiaires sans souvent savoir dans quel cadre ces stagiaires leur sont envoyés. A plusieurs reprises, il a pu être noté que les employeurs ne savaient pas si les stagiaires suivaient un stage d'insertion ou de qualification et d'ailleurs ne connaissaient pas bien les différences entre ces deux types de stage. De même, les organismes ont souvent omis de prévenir les entreprises quant aux caractéristiques des jeunes à accueillir. La mauvaise connaissance institutionnelle qu'ont les employeurs du dispositif apparaît aussi dans le fait qu'aucun des employeurs que nous avons rencontrés ne connaissait la possibilité d'imputation des $375 \mathrm{~F}$ par stagiaire et par mois sur la contribution supplémentaire de $0,1 \%$.

Il ne faut donc pas s'étonner de constater qu'au sein des vingt-cinq entreprises que nous avons enquêtées, seules deux ont signé un cahier des charges, et encore, ce fut sur leur insistance.

Les entreprises qui se sont impliquées volontairement dans le dispositif se sont révélées déçues de l'attitude des organismes de formation. Mais elles ont parfois pris l'initiative d'échanges avec ceux-ci qui ont pu conduire à une dynamique entre elles-mêmes et les organismes, qui a englobé la définition des postes de stage ou du travail du tuteur. Mais ces expériences ont été rares.

L'absence de définition quant au contenu du stage de la part de l'organisme n'a pas été sans conséquence sur le dćroulement de celui-ci.

\section{I.A RÉALITÉ DES STAGISS}

$\mathrm{Au}$ cours de ce dernier point, nous donnerons quelques informations sur la façon dont les stages en entreprise se sont déroulés, en abordant ensuite quelques éléments de bilan où nous essaierons de mettre en perspective l'utilité pédagogique de l'alternance pour les jeunes par rapport aux expériences qu'ils auront pu connaître.

\section{Le déroulement du stage}

Compte tenu de ce qui vient d'être dit, l'organisation du stage aura été du ressort même de l'entreprise, que ce soit en ce qui concerne l'accueil des stagiaires que les tâches qui leur sont affectées.

Les entreprises n'auront procédé à aucune sélection des stagiaires. La fonction des individus qui auront accueilli les stagiaires dépendra de la taille de l'entreprise. Ainsi, dans les petites et moyennes entreprises, c'est le chef 
d'entreprise ou le directeur qui accueillera le stagiaire alors que dans les plus grandes, cette tâche sera dévolue au chef du personnel ou au responsable de la formation.

En général, à cette occasion, s'effectuera une présentation d'ensemble de l'entreprise mais celle-ci occupera rarement plus d'une journée, y compris dans le cas des plus importantes, ce qui peut poser un problème quant à la fonction de découverte du monde du travail attribuée aux stages. Dans deux cas seulement, ceux de la SNCF et le la CRAM (Caisse régionale d'assurance maladie), cette découverte de l'entreprise est incluse dans une démarche pédagogique.

Si nous nous intéressons maintenant aux tuteurs, nous pouvons dire que hormis les cas déjà cités de la CRAM et de la SNCF, il n'y a pas eu de réflexion spécifique sur le rôle du tuteur dans le cadre du dispositif, ni formation particulière.

Bien souvent, le tuteur effectif sera le chef de service, le chef de rayon, voire un ouvrier hautement qualifié dans certains garages. Dans les petites entreprises, l'ensemble des salariés s'occupera du stagiaire. Et donc, l'affectation du stagiaire ne se fera pas en général sur la base de la présence d'un tuteur dans tel service mais à partir des tâches nécessaires à effectuer, c'est clair dans le cas des grands magasins, et le choix du tuteur se fera dans un second temps, se portant bien souvent sur le responsable du service en question.

En ce qui concerne les tâches effectuées par les stagiaires, elles donnent souvent lieu à une discussion préalable avec ceux-ci. Mais nous pouvons d'ores et déjà faire une remarque en ce qui concerne les différences de tâches entre les stages de qualification et d'insertion. En effet, il apparaît que sur les deux stages de qualification observés, un seul inclut véritablement le perfectionnement ou l'apprentissage d'un métier, celui de comptabilité et informatique.

Certains stages de qualification, comme le stage de tôlerie peinture dont nous avons observé les entreprises d'accueil, s'apparentent beaucoup plus à des stages d'insertion, dans la mesure où ils auront essentiellement servi aux stagiaires à s'initier aux tâches requises.

Cette différence renvoie sans doute à des pratiques différentes de la part des organismes de formation, l'organisme s'occupant du stage de comptabilité, rattaché à une chambre de commerce ayant bâti l'ensemble de son projet sur l'acquisition, pour des jeunes ayant déjà une bonne base de formation en comptabilité, d'un savoirfaire professionnel.

Dans le cadre du stage de qualification de comptabilité et informatique, les stagiaires auront pu effectuer des opérations de saisie de données bancaires, de dépouille- ment de factures..., mettant en cuvre ainsi un savoirfaire acquis à l'école.

En ce qui concerne le stage de tôlerie-peinture, les jeunes auront pu seulement découvrir le métier, les avantages et inconvénients de la tôlerie. Leur non-sélection préalable par l'organisme de formation impliquant qu'ils n'étaient pas nécessairement prêts professionnellement à cet apprentissage. Un exemple caricatural peut être donné à propos de ce jeune dont le chef d'entreprise de stage a reconnu la bonne volonté mais dont la mauvaise vue lui interdisait la profession de tôlier.

Si nous nous intéressons maintenant aux stages d'insertion, il apparaît qu'en général, les jeunes ont eu l'occasion d'occuper des postes de travail réels. C'est en particulier le cas pour le secteur de la Santé où le niveau de qualification requis des tâches effectuées n'est pas très élevé.

Ainsi, dans le secteur de la vente, les jeunes ont eu à s'occuper de la réception des marchandises, de l'étiquetage, de la mise en rayon et de la vente dans le cas des grands magasins. Dans les magasins d'alimentation, ils effectuaient les pesées, les ventes, les mises en rayon. En définitive, les jeunes ont donc occupé des tâches réelles de vendeur. En ce qui concerne la restauration, les jeunes s'occupaient souvent de la cuisine et de la plonge, ou de l'alimentation des chaînes dans le cas de la restauration rapide.

Dans le secteur de la Santé, comme d'ailleurs souvent chez les artisans, les jeunes sont utilisés comme des aides. Ainsi, dans les crèches, ils se verront confier le change, les ménages...

La directrice d'une crèche voit d'ailleurs dans ces tâches un test pour les jeunes filles qui ont souvent un désir immature de s'occuper des enfants.

Dans le cadre des entreprises enquêtées à propos des stages d'insertion, le cas de la SNCF semble être le seul qui puisse permettre la découverte d'un métier et un début d'apprentissage pouvant conduire ultérieurement à une qualification professionnelle. Mais là encore, cette expérience part d'une réflexion approfondie sur les objectifs du dispositif et peut s'appuyer sur des terrains de stages variés.

En ce qui concerne les exigences de la part des employeurs, il apparaît que seul le respect des horaires est imposé. En général, l'employeur n'impose pas de normes de productivité, "les jeunes sont là pour apprendre ».

Bien entendu, les organismes ne seront pas restés totalement extérieurs au déroulement du stage et auront conservé un contact avec les stagiaires. Mais si un suivi 
régulier a été effectivement organisé pour les stages d'insertion, il a souvent gardé un caractère superficiel qui $n$ nincluait pas une discussion pédagogique avec les responsables de l'entreprise sur les tuteurs. Quant aux stages de qualification, le suivi semble moins systématique et souvent réalisé par téléphone.

\section{Quelques éléments de bilan}

Suite à la première année de mise en place du dispositif et au recours dans ce cadre à l'alternance. il est possible de tirer quelques premiers éléments de bilan quant au fonctionnement de celle-ci. Dans un premier temps, nous évoquerons l'impact des stages en entreprise sur le comportement des jeunes lors des périodes de regroupement. Puis, dans un second temps, sera abordé le point de vue des entreprises d'accueil.

Si les formateurs ont eu quelques difficultés à intégrer l'alternance comme élément constitutif propre de leur pédagogie, il leur a bien fallu néanmoins composer avec les effets que les stages en entreprises ont pu avoir sur les jeunes, l'attitude de ceux-ci les ayant souvent déconcertés.

En général, les stagiaires ont beaucoup d'appréhension avant la première période en entreprise. Ce qui n'est pas étonnant si l'on a à l'esprit que six jeunes sur sept n'ont jamais eu d'expérience professionnelle. Les coordonnateurs parlent de trac, de peur, quelquefois d'une certaine impatience, car certains jeunes estiment ne pas avoir besoin de formation et désirent travailler immédiatement.

Les réactions à l'issue de la première période en entreprise sont de deux types. D'un côté, certains jeunes reviennent satisfaits ou enthousiasmés, de l'autre côté, un mécontentement ou une désillusion apparaît. La dernière situation peut créer d'importants problèmes de discipline.

Toutefois, il convient de relativiser ce schéma. En effet, un coordonnateur constate que beaucoup d'inhibitions disparaissent, ce qui pose d'autres problèmes de discipline...

Si le principe de l'alternance peut représenter un outil pédagogique effectif, son application néanmoins, la perception qu'en ont eu les stagiaires, traduisent une difficulté de maîtrise de la part des formateurs. Cette situation semble se révélcr notamment imputable à la nouveauté de cet instrument pour beaucoup d'entre eux.

En ce qui concerne les employeurs, le sentiment général est plutôt positif, si l'on en croit leur intention de reprendre des stagiaires puisque seuls cinq employeurs sur vingt-cinq n'envisagent pas d'accueillir à nouveau des stagiaires.
Arrêtons-nous d'ailleurs un instant sur ce groupe car il apparaît assez homogène. En effet, il est constitué d'artisans, qui avaient déjà accueillis des apprentis et qui font partie du groupe des entreprises "entraînées " évoqué précédemment. Ces artisans reprochent aux jeunes leur absence de motivation, du goût de l'effort, leur instabilité, voire leur manque d'hygiène.

Si l'on s'interroge maintenant sur les acquis professionnels auxquels ont pu conduire les périodes en entreprise, il apparaît qu'il faille distinguer selon les connaissances initiales du stagiaire et le contenu précis du stage. Ainsi, à partir des exemples relevés, il semble que l'on ne puisse opposer brutalement stages d'insertion et stages de qualification.

En réalité, lorsque le stagiaire arrive dans un stage de qualification avec un savoir de base, il peut se familiariser au geste productif et terminer le stage avec de réelles compétences professionnelles. Mais quand il s'agit de stagiaires sans formation professionnelle préalable qui sont confrontés à des tâches requérant certaine qualification précise, tôlerie, peinture, électricité..., le stage ne pourra servir qu’à une initiation.

Enfin, lorsque les qualifications exigées ne sont pas d'un niveau trop élevé, cas de la vente et de la restauration, le stagiaire aura pu apprendre le métier.

Il apparaît donc que les réalités sont diverses au sein même des stages de qualification d'une part et au sein des stages d'insertion d'autre part et qu'elles conduisent à des processus d'apprentissage et à des cursus professionnels bien différents les uns des autres. Ceci semble vouloir impliquer une discussion beaucoup plus systématique entre l'organisme de formation ct le stagiaire d'un côté et l'employeur de l'autre.

Un phénomène intéressant à signaler concerne le fait que les stages sont souvent utilisés comme périodes d'essai non pas uniquement en vue d'embauches définitives - les employeurs invoquent souvent l'absence d'offre d'emploi - mais pour effectuer les remplacements au moment des congés, six employeurs sur les vingt-cinq rencontrés recourent à cette pratique.

Si certains employeurs invoquent la lourdeur de la tâche que représente l'encadrement des stagiaires en général, ils s'accordent pour reconnaître que les jeunes « rendent service ». En outre, les bonnes relations avec les stagiaires conduisent souvent à intégrer ceux-ci, lorsqu'ils restent suffisamment longtemps, aux activités extra-professionnelles organisées dans le cadre de l'entreprise.

En outre, la plupart des employeurs estiment avoir rendu service eux aussi, aux organismes de formation.

En définitive, il apparaît que si les jeunes ont occupé des situations de travail effectives, ils ont contribué à la pro- 
duction de l'entreprise. D'un point de vue économique, la différence entre le revenu perçu par l'individu et sa contribution au produit peut se justifier par le coût de l'investissement en formation supporté par l'employeur. Un tel processus ne peut donc se justifier que s'il y a effectivement formation. Et de la manière dont s'est organisée l'alternance cette première année, il est fort à penser que la formation a été laissée à la bonne volonté des employeurs, sans qu'un contrôle ou mieux une négociation pédagogique soient assurés par l'organisme de formation. Bien souvent, et pour être volontairement provocateur, disons que l'organisme s'est contenté de confier le jeune à l'entreprise.

Dans ces conditions, le débat n'est plus de savoir de quelle manière les employeurs ont utilisé les jeunes, mais pourquoi l'alternance a peu fonctionné comme procédé de formation interactif. En effet, sans consigne, sans suivi pédagogique, on peut logiquement imaginer, et les observations le confirment, que de nombreux employeurs auront traité les jeunes comme des salariés " normaux ", à la différence près qu'ils n'avaient pas à les rémunérer. Ce qui n'est peut-être pas mauvais du point de vue de la découverte du monde du travail, mais laisse de côté la synergie potentielle entre périodes de formation théorique et de formation pratique.

En guise de conclusion, nous allons tout d'abord revenir sur les problèmes apparus lors des stages en entreprise. En premier lieu, le faible niveau des stagiaires a entraîné des déceptions parmi les entreprises qui avaient été plus ou moins entraînées à participer au dispositif. En particulier, certains stages de qualification ont dû plus servir à une initiation qu'à une véritable acquisition de savoirfaire professionnel. D'ailleurs, on peut signaler à ce propos que c'est souvent plus le niveau des connaissances professionnelles de base qui permet de distinguer le contenu des stages en entreprises que la partition insertion-qualification.

Il semble donc que de nombreux problèmes posés par le fonctionnement des stages en entreprises pouvaient être attribués aux relations insuffisantes entre organismes de formation et entreprises.
Tout d'abord, il est à noter que les organismes ont constitué peu de groupes techniques, ouverts notamment sur l'extérieur, pour l'élaboration des stages.

Ensuite, il est apparu que les employeurs connaissaient très peu les objectifs du dispositif, les différences entre les divers types de stages, les caractéristiques des jeunes qu'ils avaient à accueillir et le contenu des tâches souhaitables à leur confier, voire les avantages financiers dont ils pouvaient bénéficier. Cette information aurait dû être fournie par les organismes, théoriquement responsables des stages.

Enfin, les organismes ont répondu de façon insuffisante aux efforts de certaines entreprises, tant sur un plan qualitatif que quantitatif. Qualitativement, la réflexion de ces entreprises quant aux fonctions des tuteurs par exemple s'est trouvée en avance sur celle de plusieurs organismes. Quantitativement, on ne peut que déplorer le fait que des places de stage au sein d'entreprises qui avaient soigné l'accueil des jeunes sont restées vacantes en nombre important.

Toutes ces remarques laissent percer une insuffisance de la démarche des organismes de formation, insuffisance certainement due à l'inexpérience de certains formateurs et à leur effectif restreint, mais il s’agira à l'avenir de réfléchir à des solutions évitant que de tels phénomènes se reproduisent.

En définitive, si les entreprises ont eu tendance à organiser le travail des jeunes en fonction de leurs propres objectifs, tout en ne soumettant pas les stagiaires à des exigences particulièrement fortes, cest que, bien souvent, il n'y avait pas eu de concertation pédagogique avec les organismes de formation. Peut-être que si celleci avait existé, il y aurait eu des conflits entre les deux partenaires et que l'entreprise n'aurait pas accepté un suivi trop rapproché de la part des organismes.

On ne peut donc pas parler d'exploitation des jeunes au cours de cette première année ou si exploitation il y a pu avoir, c'est bien souvent de par un manque de concertation effective entre organismes et entreprises, concertation peut-être explosive mais dont le caractère subversif conditionne la réussite de la pédagogie de l'alternance.

Jean-Jacques PAUL chargé de recherche au CNRS, chercheur à l'IREDU 